White Paper for the Planetary Science and Astrobiology Decadal Survey 2023-2032

\title{
A Next Generation Lunar Orbiter Mission
}

August 15, 2020

\section{Authors:}

Timothy Glotch, Stony Brook University (timothy.glotch@stonybrook.edu)

Lynn Carter, University of Arizona

Brett Denevi, APL

Wes Patterson, APL

Kurt Retherford, SwRI

Ryan Watkins, PSI

Kerri Donaldson Hanna, University of Central Florida

Harald Hiesinger, University of Münster

Timothy Livengood, University of Maryland

Lillian R. Ostrach, U. S. Geological Survey

Morgan Shusterman, Arizona State University

Emerson Speyerer, Arizona State University

Carolyn van der Bogert, University of Münster

Lisa R. Gaddis, U. S. Geological Survey
Pamela Clark, JPL

Benjamin Greenhagen, APL

Noah Petro, NASA GSFC

Sarah Valencia, NASA GSFC

Joshua Cahill, APL

Catherine Elder, JPL

Georgianna Kramer, PSI

Heather Meyer, APL

Michael Poston, SwRI

Matthew Siegler, PSI

Angela Stickle, APL

Daniel Moriarty, NASA

GSFC / University of Maryland

\section{Signatories:}

Nandita Kumari, Stony Brook University

Dany Waller, Johns Hopkins University

Parvathy Prem, APL

Briony Horgan, Purdue University

Jose M. Hurtado, Jr., The University of Texas at El Paso

Maurizio Pajola, INAF-OAPD, Italy

Alice Lucchetti, INAF-OAPD, Italy

Ross A. Beyer, SETI Institute \& NASA Ames Research Center

David T. Blewett, APL

Andrew D. Horchler, Astrobotic

Cesare Grava, SwRI 


\section{Introduction}

The Moon is the scientific foundation for our knowledge of the early evolution and impact history of the terrestrial planets. Over the last decades the lunar science community has made significant progress in addressing key lunar science and exploration goals, while defining many new high-priority scientific questions regarding the formation and evolution of the Moon. On a broad scale, the last Planetary Decadal Survey defined three scientific objectives to guide studies of the inner planets and Moon. These objectives address the origins and diversity of terrestrial planets, the evolution of life on terrestrial planets, and climate processes on Earth-like planets [1]. Many of these objectives carry forward into exploration goals of a renewed lunar human exploration program, and urgently addressing these objectives will enable the rapid development of exploration plans. A large (Flagship or New Frontiers class) Next Generation Lunar Orbiter (NGLO) mission would address the last Planetary Decadal Survey objective of understanding the origin and diversity of terrestrial planets by studying the geochemistry and geology of the Moon at an unparalleled resolution compared to other lunar mission datasets. Also, NGLO would address the objective of studying the evolution of life on terrestrial planets by furthering knowledge about the composition and distribution of volatile elements on the lunar surface and better characterizing the past and present-day impact rates in the inner Solar System in order to better understand the original delivery of water to Earth. Key exploration goals, including identifying the nature and distribution of lunar volatiles (i.e., water, ice), mapping and characterizing potentially valuable lunar resources, and establishing a human presence on the Moon, also would be addressed by NGLO.

The National Research Council Scientific Context for Exploration of the Moon (SCEM) report, Next Steps-Specific Action Team (SAT), and Advancing Science of the Moon SAT (ASM-SAT) developed scientific goals explicit to the study of the Moon. The SCEM study [2] identified eight areas of scientific research to be addressed by future lunar exploration. These areas (Table 1) include understanding lunar volcanism, geologic diversity, impact processes, regolith processes, exosphere, and interior and comprise 35 prioritized science goals (cf. Table 5.1 of the SCEM report). An advanced lunar orbiter such as the NGLO, with a payload consisting of imaging

Table 1. Scientific questions addressed by instrumentation on an advanced lunar orbiter mission

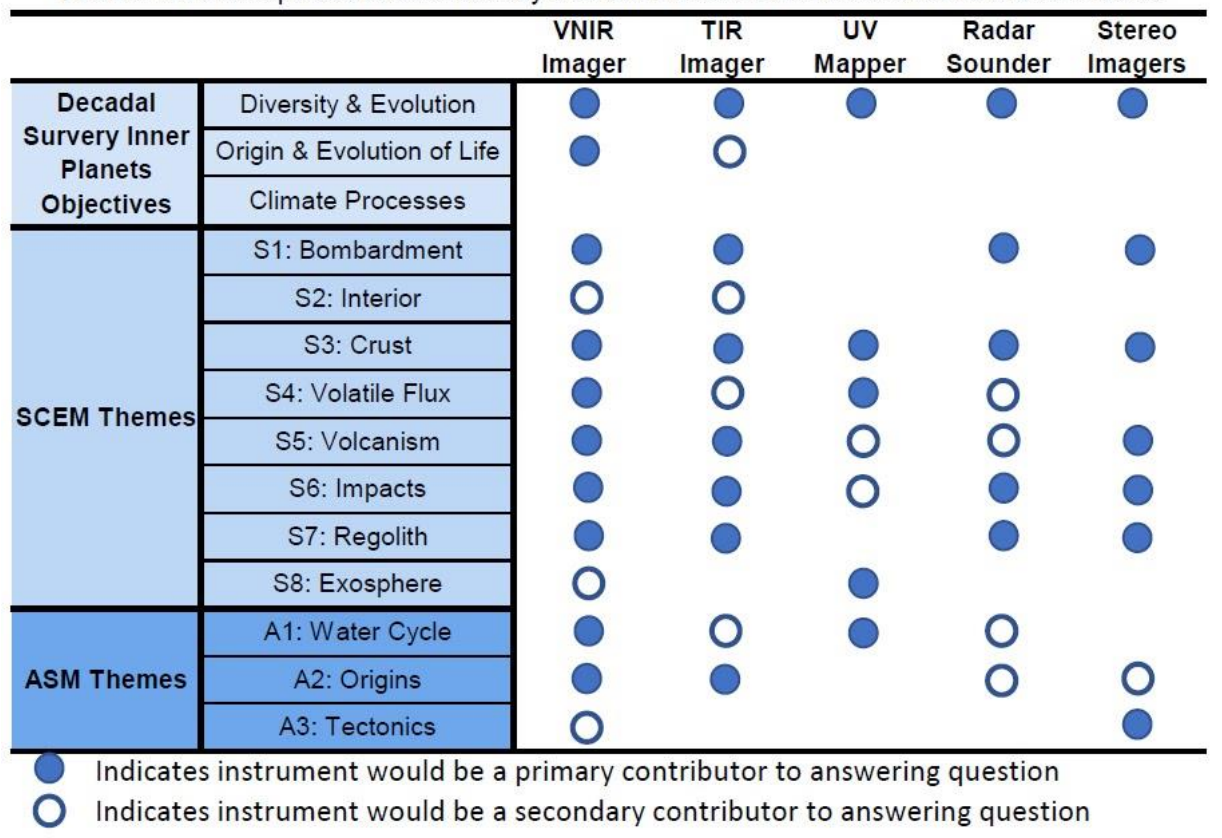

spectrometers spanning the ultraviolet (UV), visible/near-infrared (VNIR) and thermal infrared (TIR), a P-band radar sounder, and color stereo high resolution imagers (Table 2) would broadly address many of these science goals, as shown in Table 1.

In addition to evaluating the progress made in achieving the eight scientific concepts of the SCEM report (and finding that none 
of the original SCEM goals were completed), the ASM-SAT [3] also added three new concepts with additional science goals related to understanding (1) the lunar water cycle, (2) the origin of the Moon, and (3) lunar tectonism and seismicity, all of which would be addressed by NGLO.

The NGLO mission would also work toward closing lunar strategic knowledge gaps (SKGs), which were compiled by NASA's Human Spaceflight Architecture Team and two Lunar Exploration Analysis Group (LEAG) SATs. The Lunar Reconnaissance Orbiter (LRO) mission has been successful in retiring several of these SKGs, demonstrating that long-lived orbital missions are crucial for the success of future landed robotic and human missions. However, several SKGs remain open, and most require landed missions on the lunar surface and continued longlived orbital missions. The modern instrumentation, higher spatial and spectral resolution, and the

Table 2. Notional NGLO Instrument Characteristics

\begin{tabular}{|c|c|c|c|c|}
\hline Instrument & Spatial/Spectral Resolution & Data Products & Mass & Power \\
\hline $\begin{array}{l}\text { Hyperspectral } \\
\text { TIR Imager }\end{array}$ & $\begin{array}{l}\text { - } 10 \mathrm{~cm}^{-1} \text { spectral sampling } \\
\cdot 5-17 \mu \mathrm{m} \text { spectral range } \\
\text { - } 25 \mathrm{~m} / \mathrm{px} \text { spatial resolution }\end{array}$ & $\begin{array}{l}\text { Mineralogy; regolith temperature; } \\
\text { rock abundance; thermal inertia; } \\
\text { roughness; space weathering }\end{array}$ & $25 \mathrm{~kg}$ & $15 \mathrm{~W}$ \\
\hline $\begin{array}{l}\text { Hyperspectral } \\
\text { VNIR Imager }\end{array}$ & $\begin{array}{l}\cdot 7-17 \mathrm{~nm} \text { spectral sampling } \\
\cdot 0.3-5.1 \mu \mathrm{m} \text { spectral range } \\
\cdot 10 \mathrm{~m} / \mathrm{px} \text { spatial resolution } \\
\end{array}$ & $\begin{array}{l}\text { Mineralogy; volatile abundance; } \\
\text { space weathering }\end{array}$ & $37 \mathrm{~kg}$ & $22 \mathrm{~W}$ \\
\hline $\begin{array}{l}\text { UV Imaging } \\
\text { Spectrograph }\end{array}$ & $\begin{array}{l}\text { - } 115-315 \mathrm{~nm} \text { spectral range } \\
\text { - } 50 \mathrm{~m} / \mathrm{px} \text { spatial resolution }\end{array}$ & $\begin{array}{l}\text { Water ice mapping; exospheric } \\
\mathrm{OH}, \mathrm{Mg}, \mathrm{Fe} \text {, Si detection; space } \\
\text { weathering }\end{array}$ & $7.7 \mathrm{~kg}$ & $9 \mathrm{~W}$ \\
\hline $\begin{array}{l}\text { P-Band } \\
\text { Radar } \\
\text { Sounder }\end{array}$ & $\begin{array}{l}\text { - } 1 \text { m depth resolution } \\
\text { - } 4-140 \text { m sounding depth } \\
\text { - } \sim 100 \mathrm{~m} \text { along-track resolution }\end{array}$ & $\begin{array}{l}\text { Buried ice, rocks, lava flows, lava } \\
\text { tubes; ilmenite content; surface } \\
\text { roughness; heat flow (with } \\
\text { passive radiometer) }\end{array}$ & $15 \mathrm{~kg}$ & $67 \mathrm{~W}$ \\
\hline $\begin{array}{l}\text { Color Stereo } \\
\text { Imager }\end{array}$ & $\begin{array}{l}\text { - } 50 \mathrm{~cm} / \text { pixel spatial resolution } \\
\text { - } 6 \text { colors from } 350-1150 \mathrm{~nm}\end{array}$ & $\begin{array}{l}\text { High resolution multispectral } \\
\text { stereo imagery; digital elevation } \\
\text { models }\end{array}$ & $23 \mathrm{~kg}$ & $22 \mathrm{~W}$ \\
\hline
\end{tabular}

inclusion of a VNIR spectro-

meter on the NGLO mission would deliver a far more detailed and accurate lunar dataset along with the context to close remaining SKGs before and concurrent with landed missions.

\section{Science and Exploration Goals of NGLO}

The science and exploration goals of NGLO are informed by the goals and objectives outlined in the previous Decadal Survey [1], the SCEM report [2], and the ASM-SAT report [3]. The goals, objectives, and questions raised by these community-driven documents are summarized in Table 1 with a potential NGLO payload's ability to address them (Table 2). Importantly, the major strength of the mission would be the ability of NGLO to acquire simultaneous or near simultaneous observations of the lunar surface from the same platform, under consistent illumination and thermal conditions for many lunar cycles over several years. This would enable detailed mapping of surface compositions, identification and characterization of volatiles such as water and ice on the surface and in the subsurface and potential resource materials (e.g., oxygen-rich soils from pyroclastic deposits), and identification of periodic as well as transient phenomena in the lunar environment.

While landers, either robotic or crewed, and sample return missions allow for detailed characterization of local landing sites and crustal lithologies, the regional and global context required for interpretation and new discoveries of fundamentally different lithologies requires high resolution data from an orbital platform with an extended operational timeline. Numerous topics, including the past and present-day bombardment histories, the volatile cycle, and tectonic processes, are best addressed using high resolution imaging and spectroscopic data that can find and characterize the best landing sites for science and exploration activities. Further, the global datasets to be acquired by such an orbiter would permit the development of high-quality foundational products (e.g., geodetically controlled mosaics, a high-resolution 
topographic model, maps of minerals and volatiles) needed to support lunar science and in situ exploration.

2.1. Origin and Evolution of the Lunar Crust and Regolith. NGLO addresses the previous Decadal Survey goal to characterize the diversity and evolution of the terrestrial planets in the inner Solar System as well as SCEM and ASM-SAT questions related to volcanism and the formation and evolution of the lunar crust and regolith.

The lunar surface is covered by $\sim 2-10 \mathrm{~m}$ of regolith that hides many aspects of crustal geology. Radar can penetrate the regolith to characterize the near-surface stratigraphy of the Moon in unprecedented detail not available to any other orbital instrument. A P-band sounding radar $(\lambda \sim 1$ m) would allow key questions regarding the constructional history of the lunar mare to be addressed, including mapping buried subsurface flow margins [4] and searching for lava tubes, which are also of interest to the human exploration community. The radar could also be used to assess the thickness, volume, and source regions of lunar pyroclastic deposits [5]. The sounding radar system could additionally include the capability to conduct passive microwave radiometry [6], effectively producing a second instrument with the same antenna. Passive microwave sounding provides a characterization of subsurface temperatures, modulated by regolith dielectric properties. This provides a sensitivity to surface and subsurface rocks, mineralogy (such as ilmenite detection), thermal properties, and potentially ground ice. Low frequency microwave energy (300 MHz-3 GHz) could also potentially map geothermal heat flux. Such an instrument benefits from observing the entire diurnal cycle, and when combined with a surface temperature measurement, could differentiate areas where rocks or ice are found at the surface vs. only at depth.

Additional aspects of lunar volcanic history and crustal composition can be addressed by VNIR and TIR hyperspectral imagers and high-resolution color stereo imagery. Previous TIR studies have led to the discovery and characterization of highly silicic lithologies on the Moon, most of which are associated with domes [7,8], as well as global-scale geologic characterization of the bulk silicate content [9]. Silicic volcanism represents an end stage in magmatic evolution. It is important to characterize silicic features to gain a better understanding of thermal evolution, volatile-element behavior, and concentration of strategically important trace elements at these sites [8]. The limited spatial and spectral resolution of the LRO Diviner Lunar Radiometer instrument prevents more detailed characterization of the mineralogy of these sites, but photometric data from the LROC Narrow Angle Cameras (NACs) has provided additional support for evolved, highly silicic materials [10]. Multi-color photometry, as well as detailed topography and slope maps enabled by color stereo NAC-scale ( $\sim 50 \mathrm{~cm} /$ pixel) imagery, would provide further constraints on the compositions and physical properties of these enigmatic features.

To date, no intermediate (andesitic/dacitic) volcanic rocks have been identified in the Apollo sample collection or lunar meteorites, but they have been tentatively identified in remote sensing data in at least one location [10]. A high spatial resolution hyperspectral TIR imager would be the most useful tool to identify intermediate compositions and place important new constraints on both the thermal history and the formation mechanisms of evolved lunar lithologies. These constraints could support and enhance our understanding of differentiation processes and timescales on the Moon and other terrestrial bodies.

VNIR spectroscopy and spectroscopic imaging from the Kaguya Spectral Profiler instrument and the Chandrayaan-1 Moon Mineralogy Mapper (M3) provided moderate spatial and high spectral resolution views of the lunar crust, indicating a diversity of lithologies. Important detections included mapping (at $\geq 70 \mathrm{~m} / \mathrm{pixel}$ ): occurrences of the purest anorthosite (PAN) on the 
Moon [11]; olivine, much of it located in the rims of impact basins, suggesting a lower crustal or mantle origin [12]; and Mg-spinel anorthosite [13]. Unfortunately, M3 operated for just under a year in lunar orbit before failure of the Chandrayaan-1 spacecraft, and much of the data were collected under suboptimal thermal and optical conditions. A modern VNIR hyperspectral imager would enable high spatial and spectral resolution measurements of specific targets of interest including volcanic features, basin rims, and crater central peaks, which would provide new insights into the compositional variability (laterally and with depth) of the lunar crust.

The NGLO payload (Table 2) is also ideally suited to study lunar space weathering processes at a variety of spatial scales. For example, previous VNIR [14], TIR [15], UV-VIS [16], and radar [17] studies have provided new constraints on spectral and thermophysical properties of lunar swirls. Still, there remains a disconnect between our understanding of the rates and mechanisms of space weathering derived from remote spectroscopic analysis and from laboratory experiments that generate "space weathered" materials using lasers, dust impacts, or ion beams. Simultaneous or near-simultaneous high spatial and spectral resolution (Table 2) measurements of the lunar swirls that coincide with local magnetic anomalies may be key to unravelling the details of the process $[18,19]$.

2.2. The Lunar Volatile Cycle. A UV imager/spectrograph, sounding radar, and VNIR and TIR hyperspectral imagers would enable the characterization of the composition and volatile cycle of the lunar surface at unprecedented spatial scales. A long-duration mission will also characterize volatile mobility over diurnal and seasonal timescales and, potentially, decadal solar cycles. A key goal of a P-band radar sounder would be to image the lunar poles to locate and determine the depth to possible ice deposits, providing high-resolution resource maps for future robotic and human missions and constraints on the abundance, and therefore source(s) of polar volatiles. An orbital UV instrument would be capable of measuring water frost abundances at the surface in lunar permanently shadowed regions (PSRs) at $\sim 10 \mathrm{x}$ better spatial resolution than previously possible.

Direct measurements of volatiles at all latitudes would be enabled by the UV and VNIR hyperspectral imaging system, with the ability to fully characterize the $\sim 165 \mathrm{~nm}$ and $\sim 3 \mu \mathrm{m}$ $\mathrm{OH} / \mathrm{H}_{2} \mathrm{O}$ features. Coincident temperature measurements by the TIR instrument would enable high-fidelity thermal tail removal and accurate characterization of the $3 \mu \mathrm{m}$ band shape and depth. Previous measurements at UV and VNIR wavelengths have indicated spatial and diurnal variation of lunar surface hydration [20,21], suggesting a solar-wind driven hydration process.

A TIR instrument would also enable lunar volatile detection via spectral and temperature mapping methods. The combined high spectral sampling $\left(\sim 10 \mathrm{~cm}^{-1}\right)$ and range of $\sim 5-17 \mu \mathrm{m}$ of a TIR instrument would enable an assessment of multiple water $(6$ and $12 \mu \mathrm{m})$ ice absorptions. This TIR dataset would provide an independent yet highly complementary view of lunar volatiles relative to UV and NIR determinations. For example, unlike the NIR $3 \mu \mathrm{m}$ region, the $6 \mu \mathrm{m}$ water feature is free from $\mathrm{OH}$ absorption and would provide a measure of lunar hydration relative to hydroxylation with less ambiguity and greater precision. See Prem et al. [22] for additional discussion of lunar volatiles.

2.3. Characteristics of the Lunar Interior. Critical information about the composition, volatile content, and geophysics of the lunar interior would be enabled by NGLO. Several studies have utilized VNIR spectroscopy to identify apparently endogenous water content associated with crater central peaks, pyroclastic deposits, and other volcanic constructs [23,24].

NGLO's VNIR and TIR instruments would also probe the lithologies of the lower lunar crust (and perhaps mantle) by observing the compositions of crater central peaks and impact basin rims at spatial scales of 10's of meters. These measurements would be further supplemented by color 
stereo imagery at $\sim 50 \mathrm{~cm}$ spatial scales, providing the best ever spatial resolution remote compositional measurements of the lunar interior. Previous studies [25] using these techniques have been somewhat hampered by comparatively poor spatial and/or spectral resolution and lower SNR than we expect from an NGLO instrument suite.

Additionally, an NGLO radar instrument including a passive radiometer mode would provide coincident model constraints on temperatures at depth. At long wavelengths, areas found to exceed temperatures predicted from the TIR instrument would indicate the influence of a comparatively high geothermal heat flux. As heat flux increases the geothermal gradient, this difference becomes larger at longer wavelengths. For instance, the Chang'e 2 radiometer $(3 \mathrm{GHz})$ found higher than expected temperatures precisely in the areas where Lunar Prospector GRS data identified large Th anomalies, which should produce more radiogenic heat if these anomalies are representative of bulk crustal composition. The combined spatial resolutions of the radar and TIR instruments $(<100$ m) would be orders of magnitude better than GRS data. At wavelengths longer than $3 \mathrm{GHz}$, a passive instrument would be more sensitive to heat flux and less sensitive to rocks and $\mathrm{Ti}$ in the near-surface regolith.

2.4. Impacts and Bombardment History. The lunar surface provides a witness plate to both past and present bombardment of the Earth-Moon system by asteroidal and cometary debris. NGLO would continue LRO's characterization of present-day impact rates and cratering processes using high-resolution, multi-temporal imagery collected over years to decades [26]. In addition, a TIR instrument and color camera would provide high-resolution views of lunar "cold spots," which appear to form around young, small impact craters due to local regolith disturbance, and fade over time as regolith churning processes take over [27]. P-band radar would provide new information about the structure of crater ejecta and their emplacement processes [28] at higher resolution than ever before. UV, VNIR, and TIR instruments providing higher resolution compositional and mineralogical observations than previously available would give a clearer view of the diversity and distribution of impact-related materials.

2.5. Lunar Exosphere. NGLO could potentially conduct solar-occultation observations at UV, VNIR, and TIR wavelengths. Solar occultation observations can be achieved either by rolling the spacecraft or by introducing a side-looking mirror into each spectrometer with an accompanying neutral-density filter or pinhole aperture. Such observations would enable detailed spectral characterization of lofted dust scattering, lunar horizon glow, or possibly, the tenuous lunar atmosphere. These observations would also enable investigations of the exospheric response to natural meteor streams, impact probes, and landed missions.

2.6. Landing Site Characterization. The U. S. administration has proposed to land humans near the south pole of the Moon by 2024, and other nations are planning and launching landed missions as part of their lunar programs, such as the Chinese Chang'e program, the Russian Luna program, as well as potential European Space Agency (ESA) in situ resource utilization demonstration and/or human-assisted robotic sample return missions. It is clear that these missions, commercialbased missions of opportunity through NASA and ESA-supported industry collaborations, and future crewed surface missions would benefit from high-resolution landing site characterization, real-time crewed activity monitoring, and resource prospecting enabled by NGLO.

In addition to providing high-quality data for science from orbit, NGLO is ideally suited to provide high fidelity scientific and technical characterization of proposed landing sites to aid NASA and its international and commercial partners in choosing the highest science priority and safest landing sites. Color stereo imagery would provide high-resolution topography and roughness (including mapping of both slope and crater/boulder hazards) for proposed landing sites 
with subsurface ice and rock distributions from sounding radar and multi-spectral compositional information in the UV-VIS. That compositional information would be supplemented by VNIR and TIR hyperspectral imagery at spatial scales an order of magnitude or more improved over similar data sets from LRO, Kaguya, and Chandrayaan-1. Importantly, the presence of VNIR and TIR instruments making surface measurements at the same time would enable high-fidelity thermal correction of VNIR data, reducing errors and improving mapping of volatile abundances.

\section{Need for a Large Lunar Orbiter in the Age of CubeSats and SmallSats}

NASA has recently invested in cubesat and small satellite development for lunar and planetary applications. These missions will provide valuable insights into the lunar volatile cycle and lunar geology but will generally have short timescales to end-of-mission. Further, power and mass requirements for cubesat and smallsat missions necessarily exclude certain classes of instruments, often preventing the acquisition of the highest possible precision, accuracy, and spatial resolution measurements. The NGLO notional mission includes instruments with large mass or power requirements to enable high precision and accuracy measurements of the lunar surface at spatial scales up to an order of magnitude better than those available from LRO and international lunar orbiters. The resulting unprecedented, foundational data set would advance consensus lunar scientific priorities and support landed missions over the next decade or more. NGLO would enable long-term landing site scientific and hazard characterization, act as a communications relay for landed assets, and could provide quick-response, high-resolution imaging of human and robotic activity on the lunar surface. Finally, NGLO would broaden participation in lunar and planetary science and train the next generation of leaders in extraterrestrial science and exploration.

\section{Recommendation}

The development of a large lunar orbiter (a dedicated flagship mission managed through the Lunar Discovery and Exploration Program or a PI-led New Frontiers mission) with a potential lifetime of a decade or more, encouraged by the National Academies, would provide support and information that is essential to achieving near-future lunar exploration and science research goals. As the concept advances, trades between instrument suites, capabilities and observational modes, including the costs and benefits of different orbits and data acquisition plans, would need to be examined.

\section{References}

[1] National Research Council (2011), Visions and Voyages for Planetary Science in the Decade 2013-2022, Washington, D.C., National Academy Press.

[2] National Research Council (2007), The scientific context for exploration of the Moon, https://doi.org/10.17226/11954.

[3] Lunar Exploration Analysis Group LEAG (2017), Advancing science of the Moon: Report of the specific action team, held 7-8 August, 2017, Houston, TX.

[4] Campbell, B. A., et al. (2014), Improved discrimination of volcanic complexes, tectonic features, and regolith properties in Mare Serenitatis from Earth-based radar mapping, J. Geophys, Res., 119, 313-330.

[5] Carter, L. M., et al. (2009), Radar remote sensing of pyroclastic deposits in the Mare Serenitatis and Mare Vaporum Regions of the Moon, J. Geophys. Res., 114, E11004.

[6] Siegler, M. A., et al. (2020), Science case for microwave wavelength measurements, White paper submitted to the Decadal Survey.

[7] Glotch, T. D., et al., (2010), Highly silicic compositions on the Moon, Science, 329, 15101513. 
[8] Valencia, S. N., et al. (2020), End-member volcanism in the absence of plate tectonics: Silicic volcanism on the Moon, White paper submitted to the Decadal Survey.

[9] Greenhagen, B. T., et al. (2010), Global silicate mineralogy of the Moon from the Diviner Lunar Radiometer, Science, 329, 1507-1509.

[10] Clegg-Watkins, R., et al. (2017), Nonmare volcanism on the Moon: Photometric evidence for the presence of evolved silicic materials, Icarus, 285, 169-184.

[11] Ohtake, M., et al. (2009), The global distribution of pure anorthosite on the Moon, Nature, 461, 236-240.

[12] Yamamoto, S., et al. (2010), Possible mantle origin or olivine around lunar impact basins detected by SELENE, Nat. Geosci., 3, 533-536.

[13] Pieters, C. M., et al. (2011), Mg-spinel lithology: A new rock type on the lunar farside, J. Geophys. Res., 116, E00G08, doi:10.1029/2010JE003727.

[14] Kramer, G. Y., et al. (2011), Characterization of lunar swirls at Mare Ingenii: A model for space weathering at magnetic anomalies, J. Geophys. Res., 116, E04008.

[15] Glotch, T. D., et al. (2015), Formation of lunar swirls by magnetic field standoff of the solar wind, Nature Comms., 6, 6189, doi:10.1038/ncomms7189.

[16] Hendrix. A., et al. (2016), Lunar swirls: Far-UV characteristics, Icarus, 273, 68-74.

[17] Neish, C. D., et al. (2011), The surficial nature of lunar swirls as revealed by the Mini-RF instrument, Icarus, 215, 186-196.

[18] Thompson, M. S. et al. (2020), Space weathering across the solar system: Lessons from the Moon and outstanding questions, White paper submitted to the Decadal Survey.

[19] Kramer, G. Y. et al. (2020), The plethora of science afforded by a lunar swirl, White paper submitted to the Decadal Survey.

[20] Sunshine, J. M., et al. (2009), Temporal and spatial variability of lunar hydration as observed by the Deep Impact Spacecraft, Science, 326, 565-568.

[21] Hendrix, A. R., et al. (2012), The lunar far-UV albedo: Indicator of hydration and weathering, J.Geophys. Res., 117, E12001.

[22] Prem, P., et al. (2020), Lunar volatiles and solar system science, White paper submitted to the Decadal Survey.

[23] Bhattacharya, S., et al. (2013), Endogenic water on the Moon associated with non-mare silicic volcanism: implications for hydrated lunar interior, Current Science, 105, 685-691.

[24] Milliken, R. E., and S. Li (2017), Remote detection of widespread indigenous water in lunar pyroclastic deposits, Nat. Geosci., 10, 561-565.

[25] Cheek, L. C., et al. (2013), The distribution and purity of anorthosite across the Orientale basin: New perspectives from Moon Mineralogy Mapper data, J. Geophys. Res., 118, 1805-1820. [26] Cahill, J. T. et al. (2020), Assessing the present-day impact flux to the lunar surface via impact flash monitoring and its implications for sustained lunar exploration, White paper submitted to the Decadal Survey.

[26] Bandfield, J. L., et al. (2014), Lunar cold spots: Granular flow features and extensive insulating materials surrounding young craters, Icarus, 231, 221-231.

[27] Ghent, R. R., et al. (2010), Generation and emplacement of fine-grained ejecta in planetary impacts, Icarus, 209, 818-835 doi:10.1016/j.icarus.2010.05.005. 